\title{
Effects of Lentinan on Endothelial Cell Activity, Inflammatory Response, Endoplasmic Reticulum Stress, and Apoptosis in Sepsis
}

\author{
Yan Xu $\mathbb{D}$ and Yeping Du $\mathbb{D}$ \\ Emergency Department, Huai'an Second People's Hospital, Huai'an 223002, JiangSu, China \\ Correspondence should be addressed to Yeping Du; yepingdu2008@163.com
}

Received 1 January 2020; Revised 21 January 2020; Accepted 5 February 2020; Published 26 February 2020

Guest Editor: Mingqiang Li

Copyright (C) 2020 Yan Xu and Yeping Du. This is an open access article distributed under the Creative Commons Attribution License, which permits unrestricted use, distribution, and reproduction in any medium, provided the original work is properly cited.

\begin{abstract}
The aim of this study is to explore the protective effects of lentinan on endoplasmic reticulum stress, inflammation, and apoptosis in sepsis endothelial cells. Firstly, lentinan was extracted, purified, and analyzed. When the concentration of lentinan was in the range of $0.04-4 \mu \mathrm{M}$, there was no obvious effect on the morphology of HUVECs. When the concentration reached $10 \mathrm{M}$, the cells were obviously contracted and necrotic. CCK-8 cell activity experiment showed that when the concentration of lentinan reached $4 \mu \mathrm{M}$, the cell activity decreased significantly $(P<0.001)$, and it was in a dose-dependent manner. Then, the cells were divided into the control group $(0 \mu \mathrm{M}$ lentinan), sepsis group, sepsis + lentinan $1.2 \mu \mathrm{M}$ group, and sepsis + lentinan $2 \mu \mathrm{M}$ group. Enzyme-linked immunosorbent assay showed that lentinan could significantly reduce the expression of TNF- $\alpha$, IL- $1 \beta$, and IL- 6 in sepsis endothelial cells $(P<0.001)$. In addition, flow cytometry and TUNEL staining showed that compared with the control group, the apoptosis of cells in the sepsis group increased significantly $(P<0.001)$, and lentinan could inhibit apoptosis $(P<0.001)$. In terms of mechanism research, the mRNA and protein expression of endoplasmic reticulum stress-related protein in endothelial cells were detected by real-time fluorescent quantitative PCR (qPCR) and Western blotting, respectively. It was found that the expression of SIRT1, the upstream factors of endoplasmic reticulum stress in sepsis cells, was obviously inhibited $(P<0.001)$, and the expression of CHOP, GRP78, IRE1 $\alpha$, and ATF6 was significantly increased $(P<0.001)$, However, the pretreatment of lentinan could significantly reverse the above changes $(P<0.001)$. Besides, lentinan could also reduce the expression of phosphorylated p65 protein (the activation marker of NF- $k \mathrm{~b}$ ) and iNOS. Conclusion. When sepsis occurs, lentinan can protect endothelial cells from ERS inflammation and apoptosis induced by sepsis. Thus, lentinan is expected to be a new target for the treatment of sepsisinduced endothelial damage.
\end{abstract}

\section{Introduction}

Sepsis is a complex systemic inflammatory response syndrome (SIRS). It is one of the critical causes of death in patients in the intensive care unit. It can affect the heart, liver, kidney, lung, and other organ systems leading to the occurrence of multiorgan dysfunction syndrome (MODS) [1]. Although life support system technology is continuously optimized with the advancement of science and technology, the prognosis of sepsis, especially septic shock, is still very poor. The current treatment mainly involves the broad-spectrum antibiotics for infectious pathogens. However, this method largely ignores the development of sepsis, which could lead to inflammatory damage to cells and other serious complications.
When sepsis occurs, bacterial endotoxin activates monocytes to produce proinflammatory cytokines and chemokines, induces endothelial cells to transition to the proinflammatory stage, and can activate endothelial cells to produce inflammatory cytokines, such as tumor necrosis factor $\alpha$ (TNF- $\alpha$ ), interleukin $1 \beta$ (IL-1 $\beta)$, increased caspase-3 activity, thereby promoting the occurrence of apoptosis, necrosis, and other reactions in endothelial cells [2]. In addition, the release of early response cytokines can lead to the production of anti-inflammatory mediators, such as compensatory anti-inflammatory response syndrome (CARS), which can cause vascular instability and microvascular occlusion, and ultimately lead to organ dysfunction and failure [3]. In the occurrence and development of sepsis, 
SIRS and CARS are parallel to each other [4]. Therefore, effective treatment should be targeted at the early proinflammatory-anti-inflammatory stage of sepsis to prevent organ damage caused by the activation of endothelial cells.

When looking for anti-inflammatory drugs to treat sepsis, mild natural compounds caught our attention. For example, Dagmar et al. studied the effect and mechanism of Sini decoction, Baihu decoction, and Xuebi decoction in the treatment of sepsis endothelial cell model and found that the above traditional Chinese medicine has anti-inflammatory and antiapoptotic effects in sepsis [5]. It is well known that natural compounds containing $\beta$-glucan can be used to improve human health. For example, lentinan containing $1,3-\beta$-glucan is an active ingredient extracted from lentinan. Various studies have shown the anti-inflammatory, antibacterial, and anticancer potential of lentinan $[6,7]$. For example, through in vitro experiments, Nishitani et al. confirmed that lentinan can affect the expression of IL-8 and TNFR1 in intestinal epithelial cells, thereby exerting antiinflammatory activity [8]. Mizuno also reported a similar discovery that lentinan can downregulate the IL-8 mRNA expression of TNF- $\alpha$-induced Caco-2-expressing leukocytes [9]. However, in terms of sepsis research, there are few studies on lentinan, for instance, Wang et al. established a mouse model of burn sepsis and found that lentinan polysaccharides play a protective role by inhibiting the expression of inflammatory factors [10]. Therefore, this study intends to establish a model of endothelial cells with sepsis in vitro with the pretreatment of lentinan to investigate whether lentinan treatment has a protective effect on sepsis endothelial cells and explore its potential mechanism.

\section{Materials and Methods}

2.1. Extraction of Polysaccharides. Lentinan was extracted by water extraction and alcohol precipitation [11]. After the filtrate was obtained, it was concentrated, alcoholized with 95\% ethanol overnight, and dried after centrifugation to obtain the crude lentinan powder, followed by the freezethaw classification, deproteinization, and purification of the crude lentinan, and finally obtained lentinan powder [12].

2.2. Determination of Polysaccharide Content. The total sugar and reducing sugar content were measured by the phenol-sulfuric acid method and the 3, 5-dinitrosalicylic acid colorimetry, respectively. The polysaccharide content $(\%)=$ total sugar content $(\%)$ - reducing sugar content (\%) [13].

\subsection{Polysaccharide Purity Identification and Component} Analysis. Lentinan (3 mg) was dissolved in $1 \mathrm{ml}$ of deionized water, and the purity was determined by high-performance liquid chromatography (HPLC, type 1260, Agilent, USA) with SHODEX SB-806HQ column $(8.0 \mathrm{~mm} \times 300 \mathrm{~mm})$ and RID-10A parallax refractometer. In addition, gas chromatography (GC, Shimadzu, Japan) was used with a capillary column DB-1 $(0.25 \mathrm{~mm} \times 30 \mathrm{~m})$ and an FID detector to detect the components of lentinan.
2.4. Cell Culture. Primary human umbilical vein endothelial cells (HUVECs) were isolated from human umbilical veins within 10 hours after delivery. This study was approved by the ethics committee of the hospital, and the umbilical cord was provided by volunteers with signed informed consent. The umbilical vein was perfused with M199 (M199/HEPES/PS) containing 0.02 M HEPES and $100 \mathrm{mM}$ penicillin-streptomycin, and endothelial cells were obtained by digestion. HUVECs were cultured in M199 medium containing 0.02 M HEPES, 20\% fetal bovine serum, $100 \mathrm{mM}$ double antibody, $10 \mu \mathrm{g} / \mathrm{mL}$ epidermal cell growth supplement (ECGS, BD Biosciences, USA), and 15 $\mathrm{IU} / \mathrm{ml}$ heparin (Baxter, Austria). HUVECs cells between passages 4-7 were used in subsequent experiments. $\mathrm{Hu}$ man mononuclear cell line THP-1 was purchased from ATCC (USA) and cultured in RPMI-1640 medium (Sigma-Aldrich, USA) containing 10\% fetal bovine serum, $0.02 \mathrm{mM}$ HEPES, and $100 \mathrm{mM}$ double antibody at $37^{\circ} \mathrm{C}$ with $5 \% \mathrm{CO}_{2}$.

\subsection{Observation of Morphological Changes of HUVECs} Treated with Lentinan. HUVECs cells were seeded in a 24well cell culture plate and cultured to $60-80 \%$ confluency and were treated with lentinan at concentrations of $0,0.04$, $0.4,0.8,1.2,2,4$, and $10 \mu \mathrm{M} .24 \mathrm{~h}$ later cell morphology was observed with an optical microscope (Leica CRT6000, Germany).

2.6. Cell Viability Assay. Cell counting kit-8 (CCK-8, Donjindo, Japan) method was used to evaluate the proliferation activity of HUVECs. Specifically, cells were first seeded in a 96-well tissue culture plate at a density of $1 \times 10^{4}$ cells/well, and the next day, cells were treated with $0,0.04$, $0.4,0.8,1.2,2,4$, and $10 \mu \mathrm{M}$ lentinan for $24 \mathrm{~h}$. After the treatment, $10 \mu \mathrm{L}$ of CCK- 8 solvent was added to the cell culture medium for 1 hour, and the cell viability was evaluated by measuring the absorbance of the cells at $450 \mathrm{~nm}$, and the cell viability was expressed using the following formula: cell viability $=\mathrm{OD}$ value of treated cells/ average OD value of control cell $\times 100 \%$.

\subsection{Preparation of Conditioned Medium (CdM) for HUVECs} Stimulation and Lentinan Treatment. THP-1 cells were seeded into a 24-well cell culture plate at a density of $1 \times 10^{6}$ cells/well; after 10 -h culture, $10 \mathrm{ng} / \mathrm{mL}$ lipopolysaccharide was added; and the cells were cultured in the original culture environment for another $4 \mathrm{~h}$. Subsequently, the cell suspension was centrifuged at $1000 \mathrm{~g}$ for 5 minutes, and the obtained CdM was stored at $-80^{\circ} \mathrm{C}$ until use. This method of obtaining TNF- $\alpha$ by treating mononuclear cells with lipopolysaccharide has been researched and confirmed, and the method is extensively used [14]. For stimulation, HUVECs were inoculated into T25 cell culture flasks (Corning, USA) and were treated with $1.2,2 \mu \mathrm{M}$ lentinan for $24 \mathrm{~h}$. After the treatment, the cells were washed with $3 \mathrm{~mL}$ M199/HEPES/PS and then stimulated with $5 \mathrm{~mL}$ CdM for $24 \mathrm{~h}$. 
2.8. Analysis of Changes in Protein Expression with Western Blotting. Cell proteins were extracted using RIPA lysis buffer (Biyuntian, Beijing). The $\mathrm{BCA}^{\mathrm{TM}}$ Protein Detection Kit (Takara, Japan) was used to measure total protein concentration via the comparison with protein standards. A $20 \mu \mathrm{g}$ protein sample was then added to a prepared SDS-PAGE gel for electrophoretic separation. After separation, proteins were transferred to a PVDF membrane. After blocking, primary antibodies including CCAAT enhancer-binding protein (CHOP) (ab10444, Abcam, UK), glucose-regulating protein 78 (GRP78) (ab21685, Abcam, UK), inositol-requiring enzyme $1 \alpha$ (IRE1 $\alpha)$ (ab37117, Abcam, UK), activating transcription factor 6 (ATF6) (65880, cell signaling technology, UK), SIRT1 (8469, cell signaling technology, UK), NF- $\kappa$ B phosphorylation p65 (ab97726, Abcam, United Kingdom), p65 (ab16502, Abcam, United Kingdom), inducible nitric oxide synthase (iNOS) (EPR16635, Abcam, United Kingdom), and internal reference protein $\beta$-actin (ab8277, Abcam, United States) were added and incubated overnight. The next day, the primary antibody was removed, and an HRP-conjugated secondary antibody was added at room temperature for incubation. After washing, images were developed with ECL luminescent liquid (Biyuntian, China).

2.9. RNA Extraction and Measurement of $m R N A$ Expression with Real-Time Quantitative PCR ( $q P C R$ ). Total cellular RNA was extracted by TRIzol (Invitrogen, USA) method, and the cellular RNA concentration was measured spectrophotometrically. Reverse transcription into cDNA (Takara, Japan) was performed according to the instructions, and qPCR was performed by SYBR Green (Takara, Japan) method. The experimental data were analyzed by the relative Ct value method ( $2^{-\triangle \Delta \mathrm{Ct}}$ method) for relative quantitative comparative analysis, and $\beta$-actin was used as the reference gene. The target primer sequences are shown in the following Table 1.

2.10. Detection of Cytokines. Enzyme-linked immunosorbent assay (ELISA, Baosai, China) was used to determine the levels of inflammatory factors including tumor necrosis factor $\alpha$ (TNF- $\alpha)$, interleukin $1 \beta$ (IL-1 $\beta)$, and IL-6.

\subsection{Detection of Apoptosis by Flow Cytometry and TUNEL} Staining. The Annexin V (FITC)/propidium iodide (PI) method was used to detect apoptosis. Apoptosis detection kit was purchased from Beijing Baosai Biotechnology Co., Ltd. Specifically, cells were seeded at a density of $1 \times 10^{5}$ cells/well in a 6-well plate for culture. After treatment, cells were washed twice with prechilled PBS and centrifuged and resuspended in binding buffer. Then, $5 \mu \mathrm{L}$ of Annexin V-FITC solution was added and gently mixed, after which the samples were incubated for 15 minutes in the dark. Then, $5 \mu \mathrm{L}$ of the PI solution was added. Apoptosis was detected by flow cytometry (Beckman, USA). In terms of TUNEL staining, cells were treated according to TUNEL's instructions (Biyuntian, China), observed under a laser confocal microscope, and each field was randomly selected under a high-power microscope $(\times 400)$ to count the number of apoptotic cells and the total number of cells.

2.12. Statistical Analysis. Statistical analysis was performed using SPSS IBM Statistics 21 (US) software. All results were expressed as mean \pm standard deviation. $T$-test was used for comparison between two groups, and univariate analysis of variance was used for comparison between multiple groups. $P<0.05$ was considered statistically significant.

\section{Results}

3.1. Purity Determination and Component Analysis of Lentinan. The lentinan was analyzed by HPLC and showed a single narrow symmetrical peak with a relative molecular weight of about $7037 \mathrm{Da}$, see Figure 1.

The polysaccharide content of lentinan was $43.71 \%$, determined by phenol-sulfuric acid and the 3,5-dinitrosalicylic acid colorimetric methods. Gas chromatography analysis showed that it is composed of various monosaccharides including glucose, galactose, and mannose, fucose, and its molar ratio was $9.28: 3.57: 1.92: 1.03$, respectively, as shown in Figure 2.

3.2. Effects of Lentinan on HUVECs Activity. Lentinan was added to the culture medium at a final concentration of 0 (control group), 0.04, 0.4, 0.8, 1.2, 2, 4, and $10 \mu \mathrm{M}$, and cells were incubated for $24 \mathrm{~h}$. The morphological changes of the cells were observed under a light microscope. As shown in Figure 3, compared with the control group, the morphology in the lentinan treatment groups with concentrations of 0.04 , $0.4,0.8,1.2,2$, and $4 \mu \mathrm{M}$ did not change significantly, whereas the cells in the $10 \mu \mathrm{M}$ group atrophied, degenerated, and showed the state of cell death.

\subsection{Concentration-Dependent Effect of Lentinan on HUVECs} Activity. The CCK-8 assay was used to detect activity changes of HUVECs treated with different concentrations of lentinan. As shown in Figure 4, compared with the control group, when the concentration of lentinan was 0.04, 0.4, 0.8, 1.2 , and $2 \mu \mathrm{M}$, there was no significant difference in cell viability $(P>0.05)$. However, when the concentration of lentinan reached $4 \mu \mathrm{M}$, the cell viability was significantly lower than that of the control group $(P<0.001)$, which was further reduced $(P<0.001)$ when the concentration of lentinan was $10 \mu \mathrm{M}$. Therefore, in subsequent experiments, 1.2 and $2 \mu \mathrm{M}$ lentinan were used to treat cells.

3.4. Lentinan Reduces Endoplasmic Reticulum Stress Response in HUVECs with Sepsis. To investigate whether the protective effect of lentinan on sepsis endothelial cells is related to endoplasmic reticulum stress, we examined the mRNA and protein expression levels of endoplasmic reticulum stress-related factors using the qPCR and Western blotting methods, respectively. The $\mathrm{qPCR}$ results (Figure 5(a)) showed that compared with the control group, the expression of CHOP, 
TABLe 1: The primer sequences.

\begin{tabular}{|c|c|c|}
\hline Gene & Forward primers $\left(5^{\prime}-3^{\prime}\right)$ & Reverse primers $\left(5^{\prime}-3^{\prime}\right)$ \\
\hline $\mathrm{CHOP}$ & GGAAACAGAGTGGTCATTCCC & CTGCTTGAGCCGTTCATTCTC \\
\hline GRP78 & CATCACGCCGTCCTATGTCG & CGTCAAAGACCGTGTTCTCG \\
\hline IRE $1 \alpha$ & CACAGTGACGCTTCCTGAAAC & GCCATCATTAGGATCTGGGAGA \\
\hline ATF6 & TCCTCGGTCAGTGGACTCTTA & CTTGGGCTGAATTGAAGGTTTTG \\
\hline SIRT1 & TAGCCTTGTCAGATAAGGAAGGA & ACAGCTTCACAGTCAACTTTGT \\
\hline$\beta$-actin & CATGTACGTTGCTATCCAGGC & СTCCTTAATGTCACGCACGAT \\
\hline
\end{tabular}

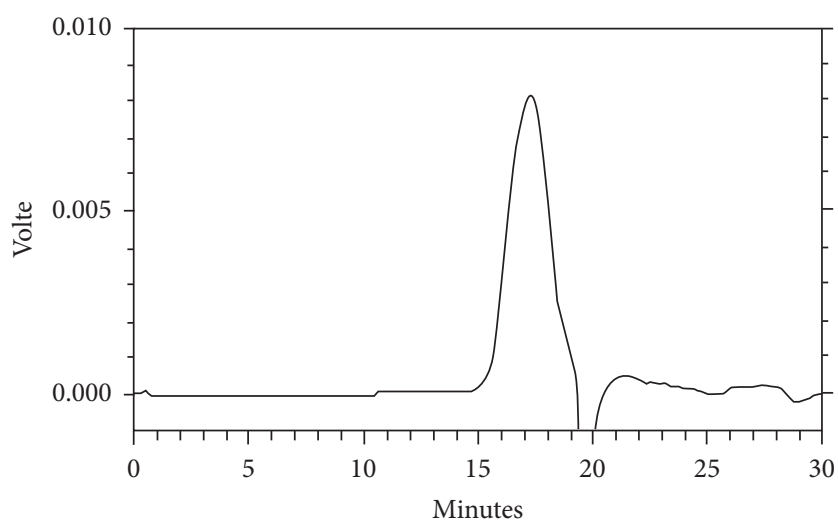

FIgURE 1: HPLC result of lentinan.

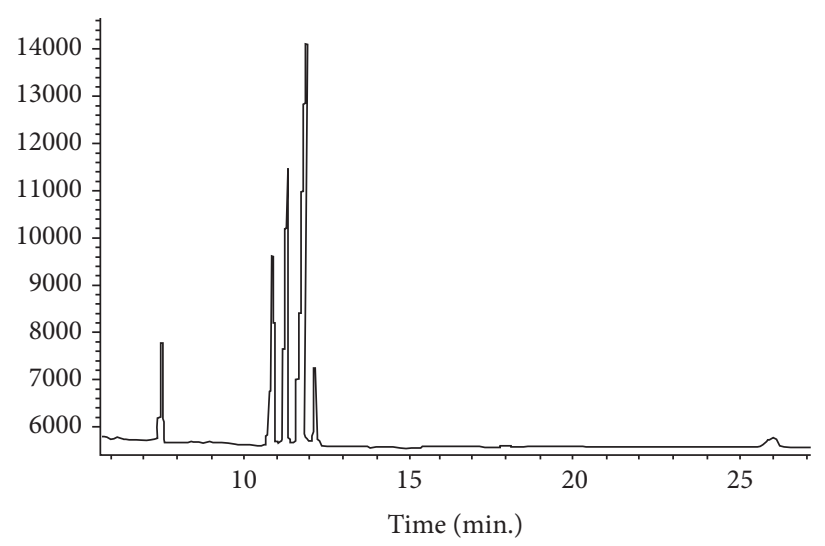

Figure 2: Gas chromatogram of lentinan.

GRP78, IRE1 $\alpha$, and ATF6 in sepsis cells was significantly increased $(P<0.001)$. However, when the cells were pretreated with 1.2 and $2 \mu \mathrm{M}$ lentinan, expression levels of these factors were significantly reduced $(P<0.001)$. The Western blotting results (Figure 5(b)) were consistent with the qPCR results $(P<0.001)$. SIRT1 is a key medium for reducing endoplasmic reticulum stress. In this study, the mRNA (Figure 5(c)) and protein (Figure 5(d)) expression levels of SIRT1 were therefore also measured. The results showed that SIRT1 expression of endothelial cells was inhibited $(P<0.001)$ in sepsis. However, when HUVECs were pretreated with 1.2 and $2 \mu \mathrm{M}$ lentinan, the inhibitory effect of SIRT1 expression was reversed $(P<0.001)$. The above results suggest that lentinan can inhibit endoplasmic reticulum stress response by upregulating SIRT1 expression in HUVECs in vitro sepsis model.
3.5. Lentinan Inhibits the Production of Inflammatory Cytokines in Sepsis Endothelial Cells. To explore the protective mechanism of lentinan on endothelial cells, we quantified the inflammatory cytokines released by HUVECs cells. The ELISA results (Figure 6(a)-6(c)) showed that compared with the control group, CdM has a stronger activation effect on the secretion of TNF- $\alpha$, IL- $1 \beta$, and IL- 6 inflammatory cytokines $(P<0.001)$. However, pretreatment with 1.2 and $2 \mu \mathrm{M}$ lentinan cells significantly inhibited the secretion of inflammatory cytokines $(P<0.001)$. In addition, we found that sepsis state can increase the expression of the inflammatory factor upstream mediator p65-phosphorylated protein and the downstream target iNOS protein, and pretreatment with lentinan can reverse this change (Figure 6(d)). 


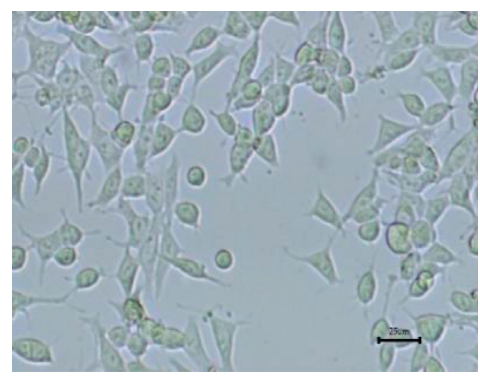

(a)

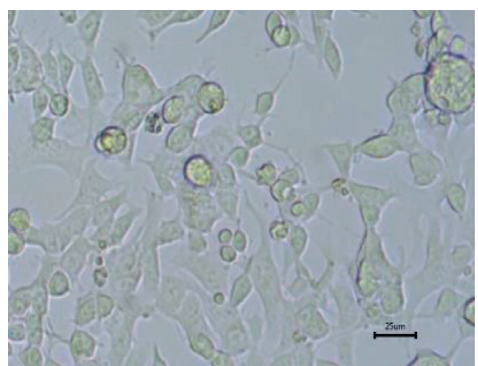

(d)

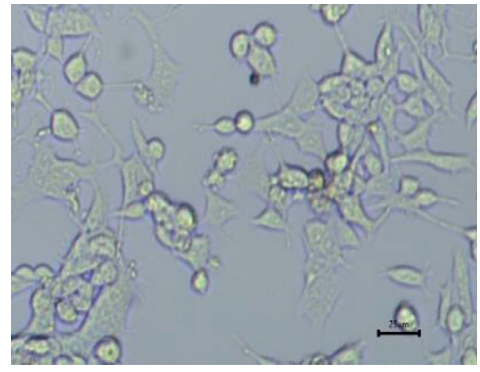

(g)

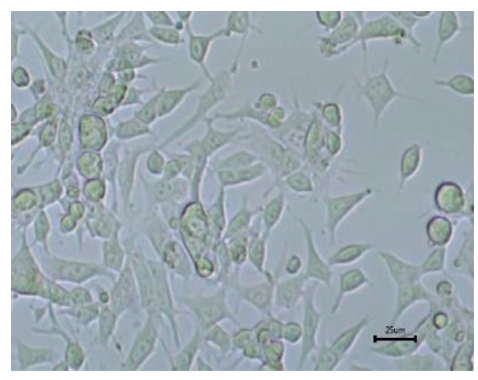

(b)

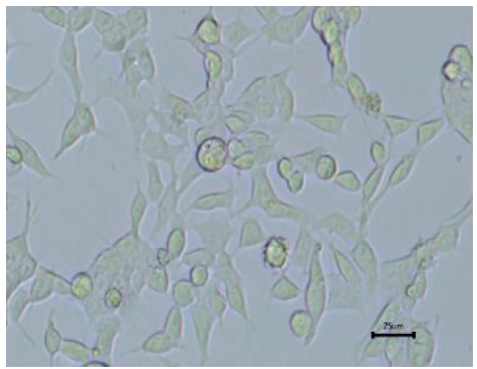

(e)

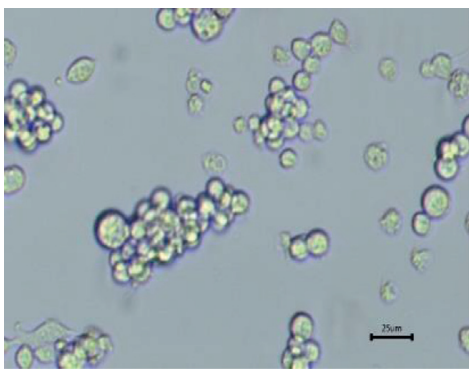

(h)

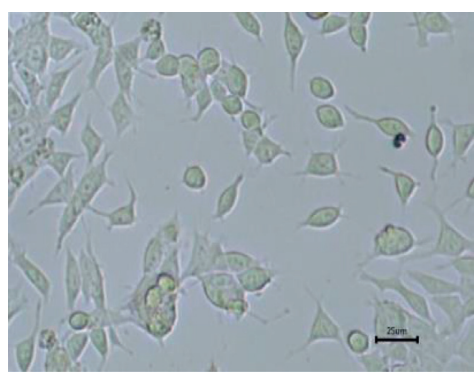

(c)

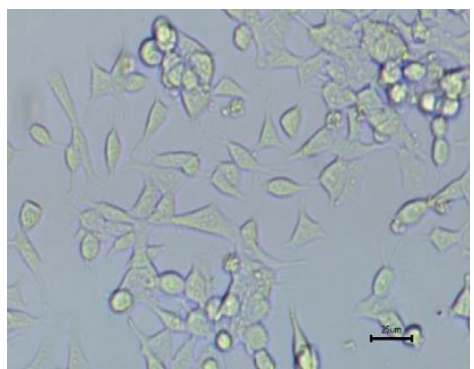

(f)

Figure 3: Concentration-dependent cytotoxic effects of lentinan on HUVECs. Figures A-H are HUVECs treated with lentinan at concentrations of $0,0.04,0.4,0.8,1.2,2,4$, and $10 \mu \mathrm{M}$, respectively. Scale bar $=25 \mu \mathrm{m}$.

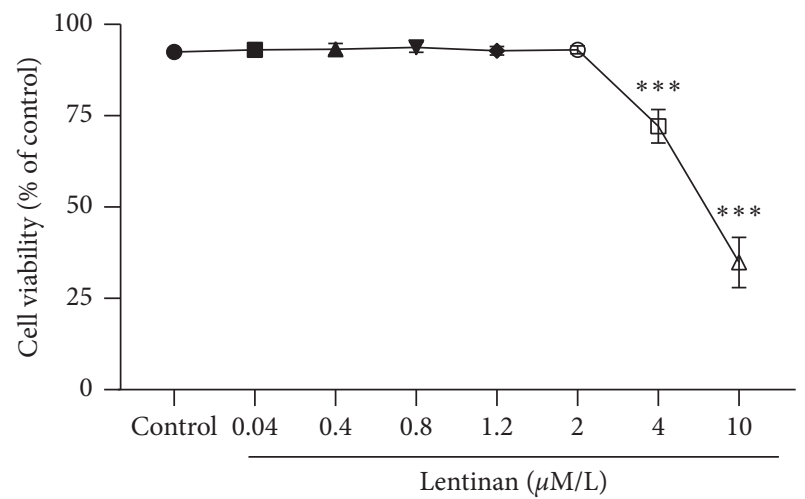

FIGURE 4: Viability of HUVECs treated with different concentrations of lentinan. Lentinan concentrations were 0 (control group), $0.04,0.4,0.8,1.2,2,4$, and $10 \mu \mathrm{M}$, and the values were expressed as mean \pm standard deviation $(n=4)$. Compared with the control group, ${ }^{* * *} P<0.001$.

3.6. Lentinan Inhibits Sepsis Endothelial Cell Apoptosis. To study the effects of lentinan on the cell apoptosis, Annexin V-FITC/PI double staining and TUNEL staining were used to detect the apoptosis of endothelial cells in the control group, sepsis group, sepsis + LNA $1.2 \mu \mathrm{M}$ group, and sepsis + LNA $2 \mu \mathrm{M}$ group. Figure 7 (a) shows that compared with the control group, the apoptotic rate of the sepsis group was significantly increased $(P<0.001)$, which was significantly reduced when 1.2 and $2 \mu \mathrm{M}$ of lentinan were administered $(P<0.001)$. Figure $7(\mathrm{~b})$ shows that the TUNEL results are consistent with the flow cytometry results $(P<0.001)$. These results suggest that lentinan can protect cells from sepsis-induced apoptotic damage.

\section{Discussion}

Endothelial cell activation and dysfunction is an important feature of sepsis and can play a key role in the development of sepsis. In this study, the HUVECs model of sepsis was established in vitro to investigate the inhibitory effect of lentinan on endothelial cell injury induced by sepsis. We found that a low concentration of lentinan had no obvious toxic effect on HUVECs. However, when the concentration of lentinan exceeded $2 \mu \mathrm{M}$, the cell viability was significantly reduced, and the cytotoxicity of lentinan was dose-dependent. We found that when HUVECs pretreated with 1.2 and 

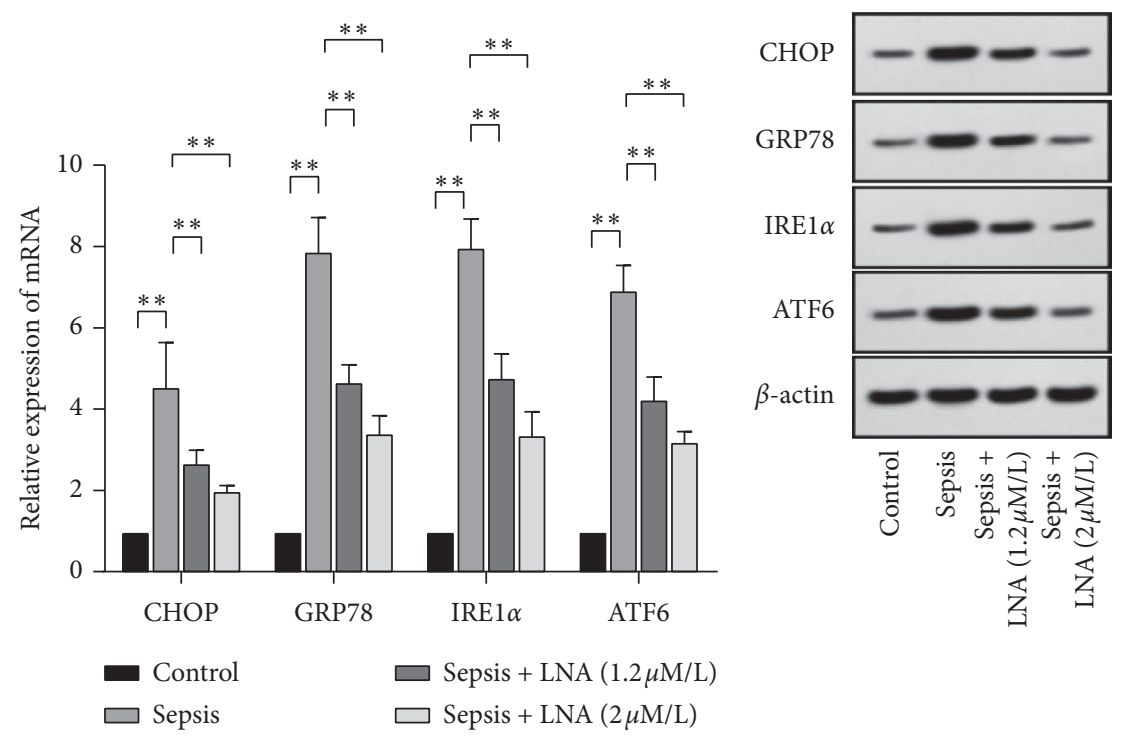

(a)

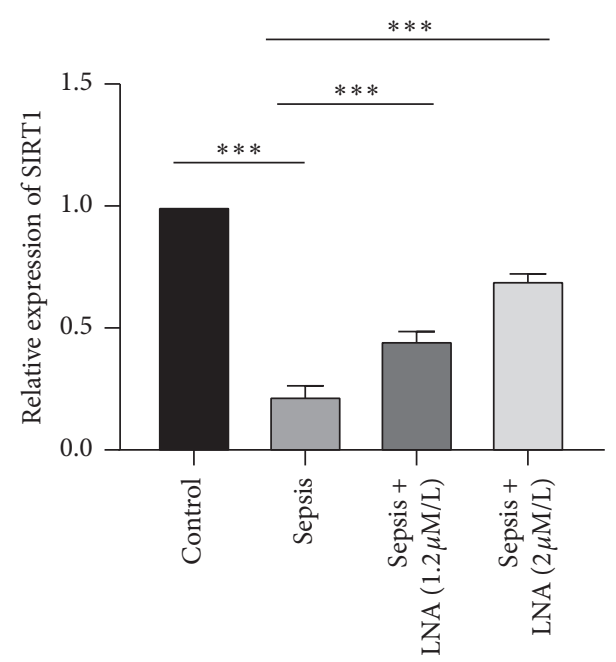

(b)

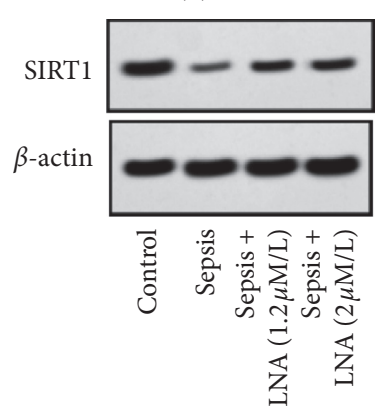

(c)

(d)

FIGURE 5: Lentinan reduces endoplasmic reticulum stress response in sepsis HUVECs. (a, b) mRNA and protein expressions of CHOP, GRP78, IRE1 $\alpha$, and ATF6 in endothelial cells of the control group, sepsis group, sepsis + LNA $1.2 \mu \mathrm{M}$ group, and sepsis + LNA $2 \mu \mathrm{M}$ group were measured by qPCR and Western blotting. (c, d) mRNA and protein expression of SIRT1 in endothelial cells of the control group, sepsis group, sepsis + LNA $1.2 \mu \mathrm{M}$ group, and sepsis + LNA $2 \mu \mathrm{M}$ group were measured by qPCR and Western blotting. Values are expressed as mean \pm standard deviation $(n=3)$. Compared with the control group or sepsis model group, ${ }^{* *} P<0.01,{ }^{* * *} P<0.001$.

$2 \mu \mathrm{M}$ lentinan and then stimulated with sepsis CdM, lentinan significantly reduced the expression of inflammatory factors such as TNF- $\alpha$, IL- $1 \beta$, and IL- 6 in sepsis, thereby reducing the inflammatory response. In addition, we found that treatment with a certain level of lentinan can inhibit endoplasmic reticulum stress in the cells; specifically, we found reduced expression of ER stress-related factors including CHOP, GRP78, IRE1 $\alpha$, and ATF6. The inhibitory effect may be due to the regulation of lentinan on SIRT1 expression. Endoplasmic reticulum stress is known to cause apoptotic responses. This study also found that lentinan can inhibit the apoptotic response of sepsis cells and reduce the inflammatory factor upstream regulatory mediator phosphorylation of $\mathrm{p} 65$ protein (NF- $\kappa \mathrm{B}$ activation marker), and downstream targets iNOS.

Sepsis is known to be a proinflammatory and anti-inflammatory coexisting complex SIRS. Endoplasmic reticulum stress plays an important role in it. Some studies have found that endoplasmic reticulum stress is involved in the occurrence and development of sepsis [15]. Endoplasmic reticulum stress can induce the release of proinflammatory factors by activating downstream signaling pathways of the inflammatory response [16], and cascade activation of inflammation further aggravates programmed cell death and 


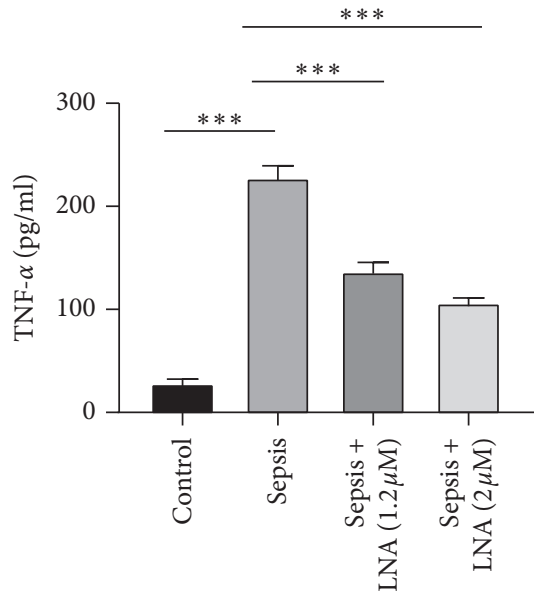

(a)

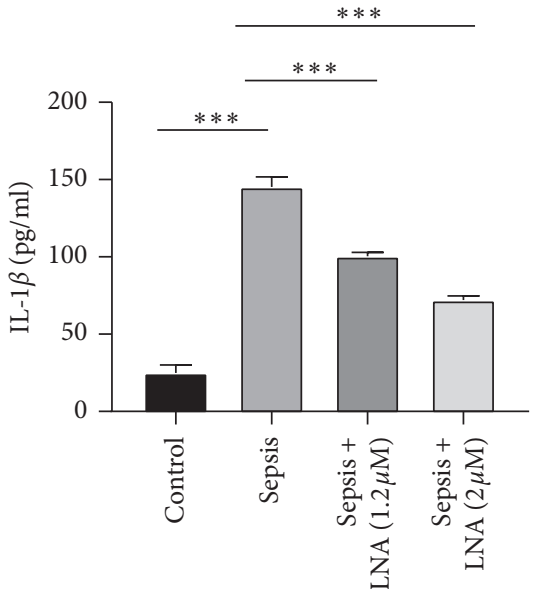

(b)

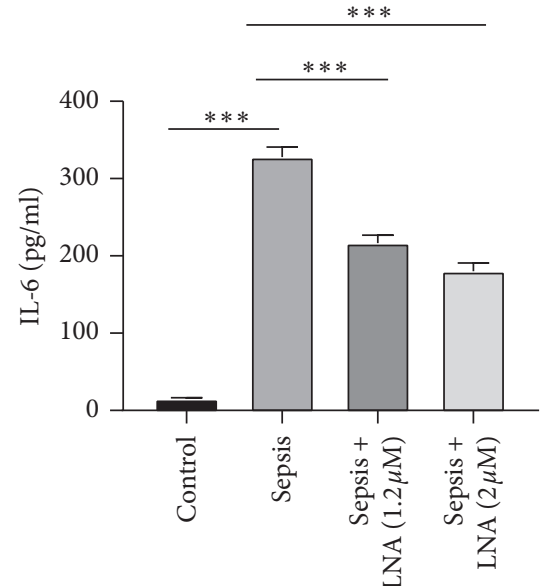

(c)

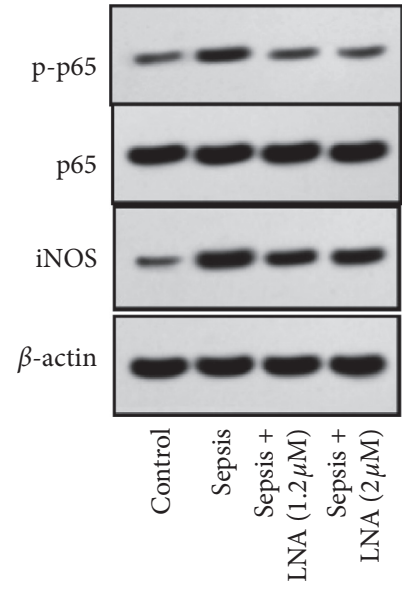

(d)

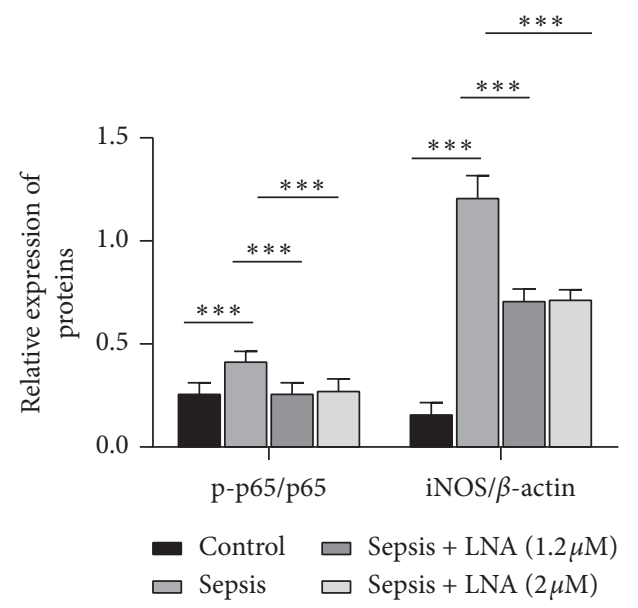

(e)

FIGURE 6: Lentinan reduces the inflammatory response of endothelial cells in sepsis. (a-c) Levels of TNF- $\alpha$, IL-1 $\beta$, and Il-6 secreted by HUVECs cells in the control group (lentinan $0 \mu \mathrm{M}$ ), sepsis group (sepsis group), sepsis + lentinan $1.2 \mu \mathrm{M}$ group (sepsis + LNA $1.2 \mu \mathrm{M}$ group), and sepsis + lentinan $2 \mu \mathrm{M}$ group (sepsis + LNA $2 \mu \mathrm{M}$ group); (d) detection of the p65 protein phosphorylation level and iNOS protein expression level by Western blotting. Values are expressed as mean \pm standard deviation $(n=4)$. Compared with the control group or the sepsis model group, ${ }^{* * *} P<0.001$.
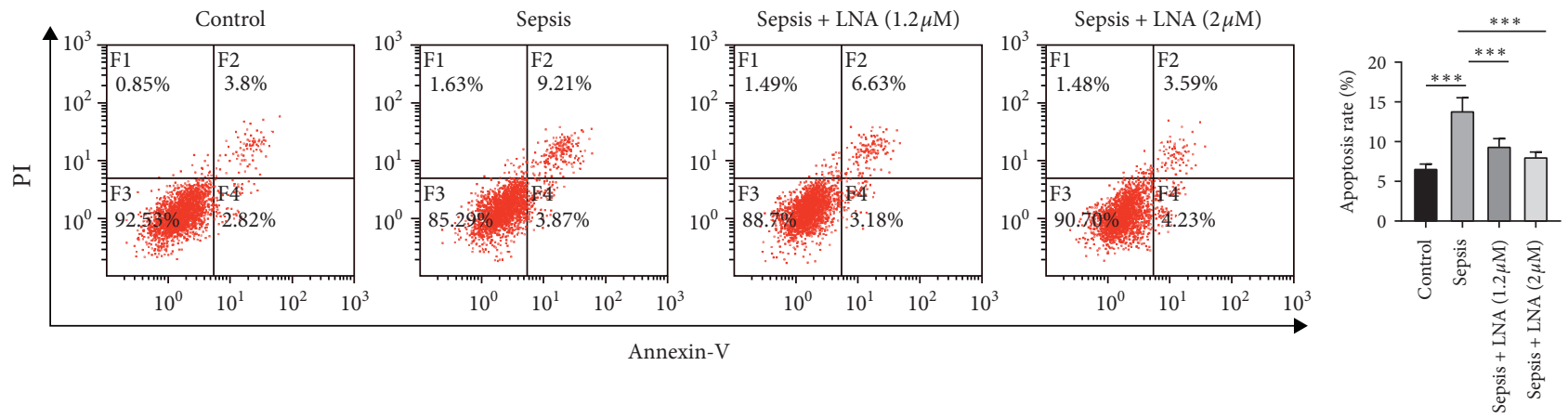

(a)

Figure 7: Continued. 

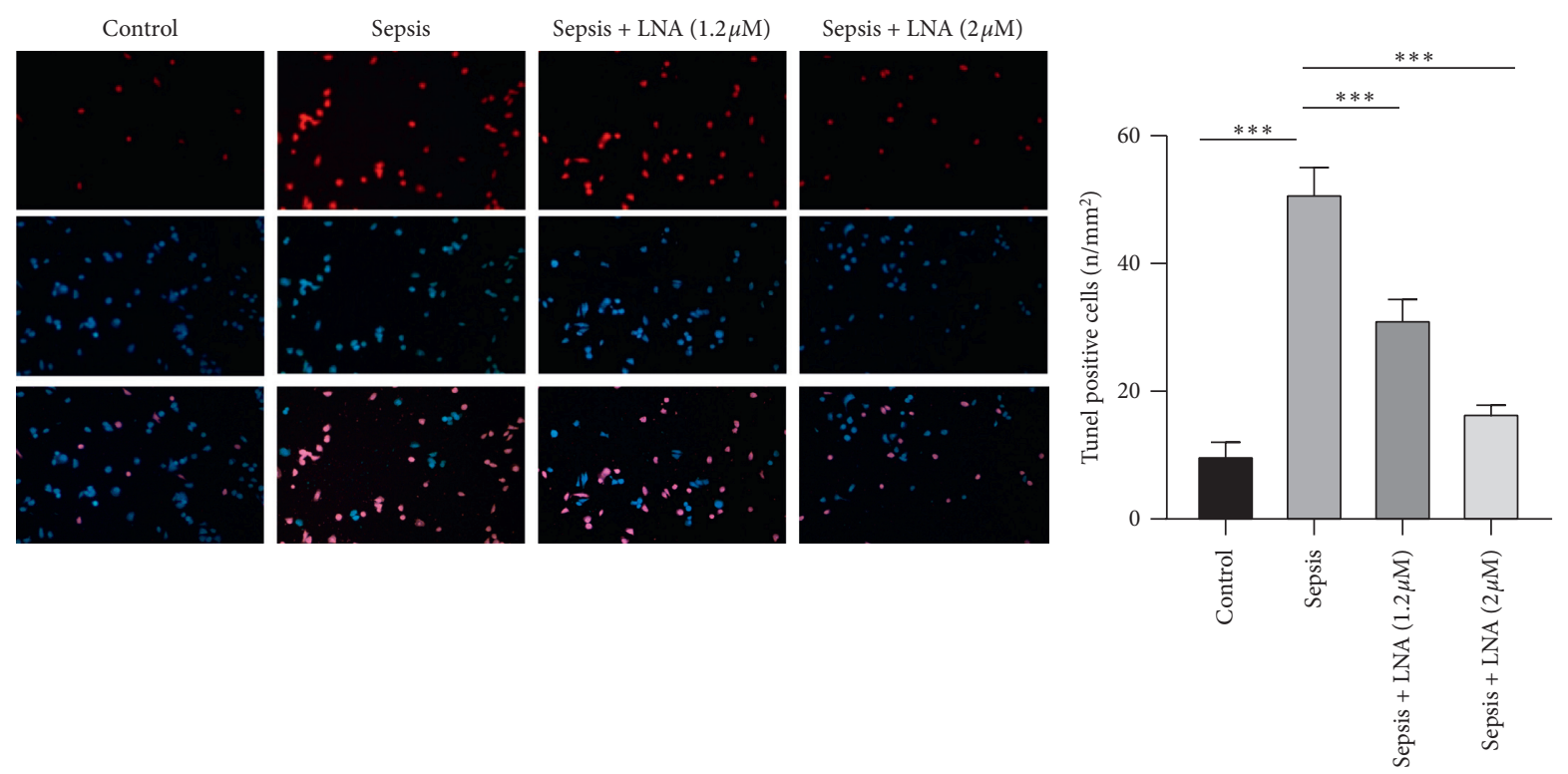

(b)

FIGURE 7: Lentinan inhibits apoptosis of endothelial cells in sepsis and expression of apoptosis-related proteins p65 and iNOS. In the control group, sepsis group, sepsis + LNA $1.2 \mu \mathrm{M}$ group, and sepsis + LNA $2 \mu \mathrm{M}$ group, (a) the flow cytometry Annexin V-FITC/PI double-labeling method was used to detect apoptosis and (b) TUNEL staining was used to detect apoptosis. Red dots denote that the nucleus stained positively by the TUNEL method, blue the nucleus of DAPI staining, while purple double staining. Values are expressed as mean \pm standard deviation $(n=3)$. Compared with the control group or sepsis model group, ${ }^{* *} P<0.01$ and ${ }^{* * *} P<0.001$.

tissue damage. Lentinan is a biological response modifier, which has the effect of reducing oxidative damage. At present, no significant adverse reaction has been reported in the human intake of lentinan [17]. Previous studies have found that lentinan can improve the immune defense system of mice by regulating inflammatory cytokines and chemokines [18]. In addition, it has been reported that lentinan can regulate the immune response by changing the structure and composition of the intestinal microbial community [18]. Zhang et al. demonstrated that lentinan can protect cardiomyocytes, inhibit apoptosis, and reduce hypoxia-induced cell damage by regulating microRNA-22/SIRT1 in primary neonatal rat cardiomyocytes [19]. However, no studies have reported the role and mechanism of lentinan in sepsis endothelial cells.

The endoplasmic reticulum stress signaling pathway is closely related to cellular inflammation, and downstream signaling pathways activated by endoplasmic reticulum stress can strongly stimulate the production of proinflammatory cytokines [20]. As important regulators of endoplasmic reticulum stress, CHOP, GRP78, IRE1 $\alpha$, and ATF6 have all been shown to play a role in regulating apoptosis [21]. Therefore, we established a model of sepsis endothelial cell in vitro, tested the expression of endoplasmic reticulum stress-related proteins under the intervention of lentinan, and demonstrated that lentinan can reduce endoplasmic reticulum stress response by inhibiting the expression of endoplasmic reticulum stress-related proteins such as CHOP, GRP78, IRE1 $\alpha$, and ATF6. In addition, as a downstream response to endoplasmic reticulum stress, the inflammatory response is an important cellular response process. Our study found that lentinan can significantly reduce the production of inflammatory factors including TNF- $\alpha$, IL- $1 \beta$, and IL- 6 , thereby reducing the inflammatory response. Furthermore, apoptosis is one of the cell outcomes caused by sepsis. In this study, flow cytometry and TUNEL staining were also used to detect apoptosis, and it was found that lentinan can significantly inhibit apoptosis and reduce the expression of the related protein.

Therefore, we believe that lentinan can protect endothelial cells and reduce damage when sepsis occurs in the body, and lentinan is expected to become a new target for treating endothelial damage caused by sepsis.

\section{Data Availability}

The data used to support the study are available from the corresponding author upon request.

\section{Conflicts of Interest}

The authors declare that there are no conflicts of interest regarding the publication of this paper.

\section{Acknowledgments}

This study was supported by the Science and Technology Funding of Huai'an (No. HAS2013008).

\section{References}

[1] L. Yang, D. Li, Y. Zhuo, S. Zhang, X. Wang, and H. Gao, "Protective role of liriodendrin in sepsis-induced acute lung injury," Inflammation, vol. 39, no. 5, pp. 1805-1813, 2016. 
[2] J. Cohen, "The immunopathogenesis of sepsis," Nature, vol. 420, no. 6917 , pp. 885-891, 2002.

[3] M. F. Osuchowski, C. Thiemermann, and D. G. Remick, "Sepsis-3 on the block," Shock, vol. 47, no. 5, pp. 658-660, 2017.

[4] A. R. Novotny, D. Reim, V. Assfalg et al., "Mixed antagonist response and sepsis severity-dependent dysbalance of proand anti-inflammatory responses at the onset of postoperative sepsis," Immunobiology, vol. 217, no. 6, pp. 616-621, 2012.

[5] D. Brislinger, C. Daxböck, E. Rossmanith, M. Stückler, I. Lang, and D. Falkenhagen, "Bai Hu Tang, Si Ni Tang, and Xue Bi Tang amplify pro-inflammatory activities and reduce apoptosis in endothelial cells in a cell culture model of sepsis," Journal of Ethnopharmacology, vol. 225, pp. 309-318, 2018.

[6] M.-F. Moradali, H. Mostafavi, S. Ghods, and G.-A. Hedjaroude, "Immunomodulating and anticancer agents in the realm of macromycetes fungi (macrofungi)," International Immunopharmacology, vol. 7, no. 6, pp. 701724, 2007.

[7] L. Ren, C. Perera, and Y. Hemar, "Antitumor activity of mushroom polysaccharides: a review," Food \& Function, vol. 3, no. 11, pp. 1118-1130, 2012.

[8] Y. Nishitani, L. Zhang, M. Yoshida et al., "Intestinal antiinflammatory activity of lentinan: influence on IL-8 and TNFR1 expression in intestinal epithelial cells," PLoS One, vol. 8, no. 4, Article ID e62441, 2013.

[9] M. Mizuno, Y. Nishitani, T. Hashimoto, and K. Kanazawa, "Different suppressive effects of fucoidan and lentinan on IL-8 mRNA expression inin vitroGut inflammation," Bioscience, Biotechnology, and Biochemistry, vol. 73, no. 10, pp. 2324-2325, 2009.

[10] C. Kupfahl, G. Geginat, and H. Hof, "Lentinan has a stimulatory effect on innate and adaptive immunity against murine listeria monocytogenes infection," International Immunopharmacology, vol. 6, no. 4, pp. 686-696, 2006.

[11] H. Zhu, K. Sheng, E. Yan, J. Qiao, and F. Lv, "Extraction, purification and antibacterial activities of a polysaccharide from spent mushroom substrate," International Journal of Biological Macromolecules, vol. 50, no. 3, pp. 840-843, 2012.

[12] Y.-j. Zhang, L.-x. Zhang, J.-f. Yang, and Z.-y. Liang, "Structure analysis of water-soluble polysaccharide CPPS3 isolated from Codonopsis pilosula," Fitoterapia, vol. 81, no. 3, pp. 157-161, 2010.

[13] Z. J. Zhang, W. Sun, L. I. Yong-Liang et al., "Determination of polysaccharide in rhizoma polygonatii by phenol-sulfuric acid and DNS methods," Chinese Journal of Experimental Traditional Medical Formulae, vol. 6, pp. 106-109, 2012.

[14] A. Schildberger, E. Rossmanith, V. Weber, and D. Falkenhagen, "Monitoring of endothelial cell activation in experimental sepsis with a two-step cell culture model," Innate Immunity, vol. 16, no. 5, pp. 278-287, 2010.

[15] M. M. Khan, W.-L. Yang, and P. Wang, "Endoplasmic reticulum stress in sepsis," Shock, vol. 44, no. 4, pp. 294-304, 2015.

[16] R. Iscimen, R. Cartin-Ceba, M. Yilmaz et al., "Risk factors for the development of acute lung injury in patients with septic shock: an observational cohort study," Critical Care Medicine, vol. 36, no. 5, pp. 1518-1522, 2008.

[17] G. Ren, K. Li, Y. Hu, M. Yu, J. Qu, and X. Xu, "Optimization of selenizing conditions for Seleno-Lentinan and its characteristics," International Journal of Biological Macromolecules, vol. 81, pp. 249-258, 2015.
[18] X. Xu and X. Zhang, "Lentinula edodes-derived polysaccharide alters the spatial structure of gut microbiota in mice," PLoS One, vol. 10, no. 1, Article ID e0115037, 2015.

[19] S. Zhang and Y. Zhao, "Lentinan protects cardiomyocytes against hypoxia-induced injury by regulation of microRNA22/Sirt1," Artificial Cells, Nanomedicine, and Biotechnology, vol. 47, no. 1, pp. 3938-3946, 2019.

[20] F. Martinon, X. Chen, A.-H. Lee, and L. H. Glimcher, "TLR activation of the transcription factor XBP1 regulates innate immune responses in macrophages," Nature Immunology, vol. 11, no. 5, pp. 411-418, 2010.

[21] R. Sano and J. C. Reed, "ER stress-induced cell death mechanisms," Biochimica et Biophysica Acta (BBA)-Molecular Cell Research, vol. 1833, no. 12, pp. 3460-3470, 2013. 